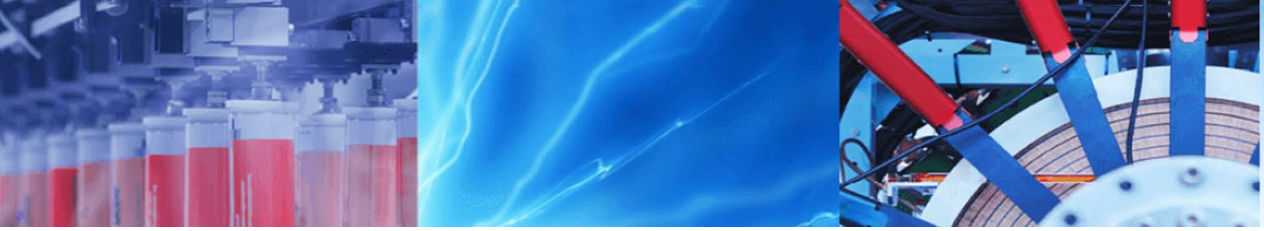

Research Article

\title{
Optimal allocation of distributed generations using hybrid technique with fuzzy logic controller radial distribution system
}

\author{
Rajesh Kumar Samala ${ }^{1}$ (D) - Mercy Rosalina Kotapuri ${ }^{1}$
}

Received: 10 November 2019 / Accepted: 3 January 2020 / Published online: 10 January 2020

(c) Springer Nature Switzerland AG 2020

\begin{abstract}
In this concept, an enhanced idea is proposed for solving the optimal load flow issues with uncertainties. In this article the Fuzzy Logic Controller (FLC) technique and Ant-Lion Optimization Algorithm's (ALOA) with Particle Swarm Optimization (PSO) based combination is proposed. The ALO imitates the hunting mechanism of ant lions in nature and the PSO improves the ALO performance by updating elitism phase of ALO. The FLC is trained based on training dataset and testing time which produces the optimal allocation parameters are based on the variation of radial distribution network parameters. In this projected hybrid algorithm, Photo-Voltaic and Wind Turbine generations (PV and WT) are considering as Distributed Generators (DGs). Initially, after defining the multi objective function, then about voltage deviation, minimization of power loss and improvement of voltage stability index is discussed. The minimization of cost of operation and deviation of voltage indexes are considered as multi objective functions and the projected technique is evaluate on IEEE 33 standard radial distribution systems. With new hybrid technique, allocation of multi-DGs like wind and PV at different sites, and the optimal load flow at various cases is analyzed.
\end{abstract}

Keywords Distributed Generations · Ant-lion optimization (ALO) · Particle swarm optimization (PSO) · Fuzzy logic controller $(\mathrm{FLC}) \cdot$ Voltage stability index $(\mathrm{VSI}) \cdot$ Operational cost and power loss reduction

\section{Introduction}

Evolution of various methodologies had been discussed by worldwide to associate the grid and electric power market deregulation [1]. The power loss during the transmission from large central power plant is compensated by small-scale power station with DG at load centers [2]. With the assist of green energy majority of the DG energy sources are regarded that is presumed pollution free. DG innovations have different technical merits. Gas turbine, wind, geothermal, photovoltaic, and biomasses are various types of DGs. Moreover, DGs are available in modular units, and regarded by ease of determining locations to, shorter construction times, and small generators and small initial cost [3]. Improvement in voltage, power loss reduction, power quality enhancement, and enhanced utility system reliabilities are the technical advantages with DG. By establishing DG at right position with proper size, augmented power losses are reduced [4].

The converter is providing real power to local needs and supplying reactive power for voltage stabilization [5]. While, with DG type will have a significant solution on power system quality. Unsuitable site will lead to rise in network losses and sometimes it might even shutdown the total network [6]. At the distribution side there is a higher interest in penetration of DG since of major issues such as minimization of power loss, environment friendly, minimization of cost, and improvement of voltage [7]. At the distribution side there is a higher interest in penetration of DG because minimization of power loss,

Rajesh Kumar Samala, rajeshkumar_samala@yahoo.co.in | 'Department of Electrical and Electronics Engineering, Vignan's Foundation for Science, Technology and Research (Deemed to be University), Guntur, Vadlamudi, India. 
environment friendly, minimization of cost, and improvement of voltage will done by DG [8].

Several accesses have been considered for sizing of the DG and optimal sitting by economical performances and dissimilar optimization method enlightening technically [9]. Methods specified as, hybrid methods, heuristic, and deterministic are predicting and even evolving in this area. Settling a DG, minimization of power loss, may be the base solution of Tabu Search (TS), direct search algorithm, PSO algorithm and Ant Colony Optimization Algorithm (ACOA) algorithms. Predictably, the mixed integer linear program, Genetic Algorithm (GA), is applied to inquire the optimal placement and sizing of DG. Although laying the DGs emphasis in everyone's articles, was rendered on the decreasing of line loss mostly. System voltage also has examined to buildup [10]. Accordingly, deregulation of power industry will be employed by the optimization methods, the best assignation of the DG will be allowed, an effective algorithm is to inspect the load flow effect and the location trouble of DG will be suggested by this article. In this research, the optimal allocation problem was solved with proposed hybrid FLC and ALO algorithm. The power loss reduction, operational cost, deviation of voltage index and voltage stability indices are the main objective functions in this article. Literature on this research work is presented in Sect. 2. Next, in Sect. 3 presented mathematical problem formulation and objective functions. The detailed explanation of the proposed technique is presented in Sect. 4. Then, in Sect. 5 presented all the outcomes of this research and finally the conclusion part is presented in Sect. 6.

\section{Recent research work: a brief review}

Here this literature presents about the research work has done previously on optimal sizing and location of DG in power scheme. Some of the works were reexamined here.

The long-term procedure characteristics of distributed generation have been proposed by Wang et al. [11]. To determine the measuring of Soft open points and sitting (SOPs) efficiently was carried by a mixed integer non-linear optimization consequence, on the means of stimulated by Wasserstein distance will be stimulated by the typical operation scenarios. The power electronic devices are the SOPs, corresponded to exchange open points in active electrical distribution strategies. SOPs should establish the regulation of voltage along usual operating conditions and real and/or reactive load flow control and fast fault separation and provide repair along irregular conditions. To evolution the distribution controllability strategies, progress augment, flexibility, and economy of the framework is the SOP's main function.
A designing pattern for electrical distribution companies (DISCOs) to optimize profits have been invented by Mokgonyana et al. [12]. Optimal network location and capacity will be determined by the example for non-conventional energy resource, as independent production of power (IPP) and Self Generation (SG) were conceived. Sources will be concerned by IPP possessed by investors of third-party and associated to a share responsibility arrangement. Shorter generators were admitted by SG are improved by feed-in tariffs, that concede local consumption for energy, producing surplus generation to the distribution system. Evaluate network capacity was allowed to produced optimal planning model optimize earnings if the DISCO is fulfilled to establish network approach to IPP and SG. The accusative purpose had a distinguishable parts, the resolution of SG is deserve, are recovery, revenue loss, and also the excess energy cost.

Selam et al. [13] have presented the work of an allocation of Distributed Generation (DG) units and Distribution Static Synchronous Compensator (D-STATCOM) by proposing an analytical and meta-heuristic hybridization algorithm for power loss minimization in distribution network. In this research, by considering Voltage Stability Index (VSI), developed a hybrid method with analytical and a Sine-Cosine Approach (SCA). The selection of optimal site for DG integration was done based on the VSI and the optimal size of D-STATCOM and DG was evaluated by SCA. This research was validated with the existing techniques and is executed on IEEE-12 and IEEE-69 standard bus systems using MATLAB.

Ali et al. [14] has presented by Ant Lion Optimization Approach (ALOA) for optimal allocation of DGs with nonconventional sources for different distribution systems. Thus, WT and PV has taken as sources of Distributed Generation (DG). Initially, vary weak buses are identified with Loss Sensitivity Factors (LSFs) for locating DGs. Now the projected ALO was utilized to evaluate the allocation of DGs from selected buses.

For voltage backup the Battery Energy Storage (BES) in distribution side with incorporating solar had been invented by Babacan et al. [15]. Minimizes the voltage fluctuations will be produced by a GA based bi-level optimization technique done by PV expansion by spreading BESS between distribution network acceptable nodes while describing for their capital, installation costs and land-ofuse applying a qualitative cost model. BESS capacity and installation points will be evaluated by the optimization problem and the distribution system as conclusion parameters. Linear programming (LP) function will be examined by BESS methodology and that reduces the daily coincident peak demand.

El-Ela et al. [16] has developed a Water Cycle Algorithm (WCA) for optimal allocation of DGs and Capacitor Banks 
(CBs). This projected technique was to get technical, financial, and environmental advantages. Various objective functions: power loss minimizing, minimization of voltage deviation, cost of electrical energy, production of emissions by sources of generation and enhancement of the voltage stability index were considered. WCA imitates the flow of water cycle from streams to rivers and to sea.

Samala et al. [17] has done the power loss comparison in radial system after and before DG integration. For comparative analysis the conventional Bat algorithm (BA) and Gravitational Search Algorithms (GSA) has been considered. To determine the initial power loss at base case without DG integration was done by using Backward/Forward Sweep approach. Then by using BA and GSA algorithm the optimal location and capacity of DGs were investigated and the minimization of real power loss has been examined. The Photo-Voltaic based energy is considered as DG in this work. The research has been carried on IEEE-33 radial test system by using MATLAB software.

\section{Problem formulations and objectives}

Here, the issue is about optimal allocation of multiple DGs with different power factors in distribution systems. Initially, the Wind turbine (WT), PV system based DG characteristics are analyzed and evaluated Voltage Stability Index (VSI) of IEEE-33 standard test system. Based on VSI data, the numbers of sites are determined for achieving the optimal allocation and sizing. Before that, the instability in output power should be balanced by the combination of all generator outputs and reserve. Generally, the proper allocations are determined including the power loss, power factor and cost of WT, PV system based DGs. The multi objective solution is evaluated from the following equations,

$\mathrm{Z}_{F}=\operatorname{Min}\left(\psi_{1}+\psi_{2}+\psi_{3}+\psi_{4}\right)$

Where, $Z_{F}$ is denoted as the multi-objective function. The parameter $\psi_{1}, \psi_{2}, \psi_{3}$ and $\psi_{4}$ is denoted as the power loss, operational cost, voltage deviation index, and voltage stability index. Here, this objective function should be minimized to obtain the optimal allocation of WT, PV based DG.

\subsection{Power loss reduction}

The power loss minimization done by wind, PV based DG allocation was measured as the ratio of before DG total power loss to after WT, PV based DG allocation in the network and it is represented by [18],
$\psi_{1}=\Delta \mathrm{PL}_{P V, W T}=\frac{P_{D G, T L O s s}}{P_{T L o s s}}$

Where,

$P_{\text {TLoss }}=\sum_{m=1}^{n b} P_{\text {Loss }}(m, m+1)$

Here $n b$ is total number of branches. The net power loss reduced with wind and PV based DG location in the network will be maximized by minimization of $\triangle P L_{P V, W T}$.

\subsection{Minimization of operational cost}

The cost of operation of DISCOs has two components. Very first cost is for active power supplied from substation. This will be reduced by reducing the total system power losses [18]. The next is the cost of active power supplied by the WT, PV based DGs connected. This cost will be reduced by reducing the quantity of active power supplied by DGs. So the minimization of Total Operating Cost (TOC) is given as [18].

$\min (T O C)=\left(\alpha_{1} P^{T L O s S}\right)+\left(\alpha_{2} P_{P V D G}^{T}\right)+\left(\alpha_{3} P_{\text {WTDG }}^{T}\right)$

Where, $a_{1}, a_{2}$, and $a_{3}$ are the coefficients of active power supplied by substation and WT, PV based DGs in $\$ / \mathrm{kW}$. $P_{P V D G}^{T}$ and $P_{W T D G}^{T}$ is the total real power drawn from connected PV, WT based DGs. PPVDG and $P_{W T D G}^{\text {TLoss }}$ is the total system power loss. The total operating cost $(\triangle \mathrm{OC})$ of PV, WT reduced is calculated as [18],

$\psi_{2}=\Delta O C=\frac{T O C}{\alpha_{3} P_{P V D G}^{\max , T}}+\frac{T O C}{\alpha_{4} P_{W T D G}^{\max , T}}$

The system TOC with DG connection can be reduced by reducing $\triangle O C$.

\subsection{Voltage deviation index (VDI)}

Bus voltage maintenance within the constraints is the most significant issue. A small difference in the voltage disturbs the entire network and might be leads to block out situation. The voltage which diverges from rated value of voltage is represented as [18],

$\psi_{3}=V D I=\sum_{i}^{N} \frac{\left|V_{\text {rated }-V_{i}}\right|}{V_{\text {rated }}}$

Where, $V_{\text {rated }}$ is the system rated voltage would be 1.0 p.u. $V_{i}$ is the ith bus voltage in p.u. $\mathrm{N}$ is the system total number of buses. 


\subsection{Voltage stability index}

In radial distribution network voltage stability index is represented by [19],

$$
\begin{array}{r}
S I\left(n_{i}\right)=\left|V_{m i}\right|^{4}-4\left[P_{n i}\left(n_{i}\right) \cdot R_{n i}+Q_{n i}\left(n_{i}\right) \cdot X_{n i}\right] *\left|V_{m i}\right|^{2} \\
-4\left[P_{n i}\left(n_{i}\right) \cdot R_{n i}+Q_{n i}\left(n_{i}\right) \cdot X_{n i}\right]^{2}
\end{array}
$$

The objective function to improve voltage stability index is [19],

$\psi_{4}=\left(\frac{1}{\operatorname{Si}\left(n_{i}\right)}\right) n_{i}=2,3, \ldots, n_{n}$

Where, $V_{m i}$ is the voltage of bus $m_{i}, P_{n i}\left(n_{i}\right)$ is the total active power load injected through bus $n_{i}, Q_{n i}\left(n_{i}\right)$ is the total reactive power load injected through bus $n_{i}, R_{n i}$ is the reactance of branch $i$, the reactance of branch $i$ is denoted by $X_{n i} \cdot P_{n i}$ and $Q_{n i}$ are any bus $n_{i}$ is obtained from the Eqs. (9a) and $9 \mathrm{~b}[19]$

$P_{n i}\left(n_{i}\right)-j Q_{n i}\left(n_{i}\right)=V_{n i}^{*} I_{n i}$

Here,

$I_{n i}=\frac{V_{m i}-V_{n i}}{R_{n i}-j X_{n i}}$

$I_{n i}$ is the branch current of the radial system, $V_{n i}$ is the voltage of bus $n_{i}$ and $V_{m i}$ and $V_{n i}$ is the voltage of bus $m_{i}$ and $n_{i}$ respectively. For the optimal allocation and sizing analysis, the following constraints are defined and utilized.

\subsection{Constraints}

DG limits and line flow limits are have their own minimum and maximum limits and mathematically given by [19],

$$
\begin{aligned}
& P_{S S}=\sum_{m=2}^{n} P_{L m}+\sum_{m=1}^{n b} P_{L o s s}(m, m+1)-\sum_{m=1}^{n b} P_{C a p, m} \\
& P_{c m}^{\min } \leq P_{c m} \leq P_{c m}^{\max } m=1,2, \ldots, n_{b} \\
& Q_{c m}^{\min } \leq Q_{c m} \leq Q_{c m}^{\max } m=1,2, \ldots, n_{b} \\
& P_{D G}^{\min } \leq P_{D G}(i) \leq P_{D G}^{\max } \\
& Q_{D G}^{\min } \leq Q_{D G}(i) \leq Q_{D G}^{\max }
\end{aligned}
$$

$S_{L i} \leq S_{L i(\text { rated })}$
Where, $P_{c m}^{\min }$ and $Q_{c m}^{\min }$ are the minimum reactive and active power limits of compensated bus $m . P_{D G}^{\min }$ and $P_{D G}^{\max }$ are the lower and upper limits of active outputs $D G s, Q_{D G}^{\min }$ and $Q_{D G}^{\max }$ are the lower and upper limits of reactive outputs DGs, $S_{L i}$ is the complex power in line $i$ and $S_{L i(\text { rated })}$ is the rated complex power in that line $i$ respectively.

\subsection{Power flow equations of DGs}

In power flow studies DGs will be modeled as PV or PQ buses. Compared with conventional power plants the DGs voltage regulation capability is limited as they are smallcapacity units. DG might reach their limits of reactive power and the PV bus changes into a PQ bus if it is modeled as PV bus [20]. In an optimal allocation problem DGs were considered as negative loads. In this research DG output is a source of constant reactive and active power generation.

Here $m$ is source node and $m+1$ is destination node [21]. From the Fig. 1 reactive and active powers are measured by utilizing the following of Eqs. (16) and (17) [21]:

$P_{m+1}=P_{m}-P_{L(m+1)}-R_{m, m+1} * \frac{\left(P_{m}^{2}+Q_{m}^{2}\right)}{\left|V_{m}\right|^{2}}$

$Q_{m+1}=Q_{m}-Q_{L(m+1)}-X_{m, m+1} * \frac{\left(P_{m}^{2}+Q_{m}^{2}\right)}{\left|V_{m}\right|^{2}}$

Line voltage is calculated using Eq. (18) [21]:

$$
\begin{aligned}
\left|V_{m+1}\right|^{2}= & \left|V_{m}\right|^{2}-2\left(R_{m, m+1} \cdot P_{M}+X_{m, m+1} \cdot Q_{m}\right) \\
& +\left(R_{m, m+1}^{2}+X_{m, m+1}^{2}\right) \cdot \frac{\left(P_{m}^{2}+Q_{m}^{2}\right)}{\left|V_{m}\right|^{2}}
\end{aligned}
$$

Actual power loss of the system is determined with Eq. (19) [21]:

$P_{\text {Loss }}(m, m+1)=R_{m, m+1} * \frac{\left(P_{m}^{2}+Q_{m}^{2}\right)}{\left|V_{m}\right|^{2}}$

Entire power loss of the network is obtained by adding total losses in the line given in expression (20) [21].

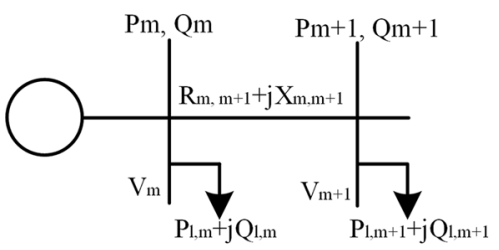

Fig. 1 General radial distribution system 
$P_{\text {TotalLoss }}=\sum_{m=1}^{n-1} P_{\text {Loss }}(m, m+1)$

Here, from bus $m$ the reactive and real power flows are $Q_{m}$ and $P_{m}$, reactive and real power demand at bus $m, Q_{L(m+1)}$ and $P_{L(m+1)}$, reactive and real power demand at bus $m+1, R_{m, m+1}$ is resistance connected between buses $m$ and $m+1, X_{m, m+1}$ is the reactance connected between the buses $m$ and $m+1$ and $V_{m}$ is the voltage at bus $m$. DGs are categorized into various types and to validate results proposed hybrid technique is applied to the first three types [22]. In the paper, the WT, PV system is considered as a DG. For locating the maximum power loss location FLC based ALO-PSO is used and the optimal DGs are placed. From next part, detailed study of projected hybrid methodology based optimal allocation and sizing with Wind Turbine and PV based DGs troubles are described.

\section{Proposed hybrid technique for multiple PV and wind turbine based DGs allocation}

In the paper, the proposed hybrid technique is utilized for solving the optimal allocation and sizing issues. To analyze the optimal allocation and sizing problems, the multi objective function are defined a radial distribution system. Initially, the normal line power flow is analyzed after that, the corresponding real and reactive power capability is determined. During the uncertainty period, the generators are considered as PV and WT placement of optimal allocation and sizing. For that analysis, the power loss reduction, minimization of operational cost, minimization of voltage deviation index and improving voltage stability index are determined with help of the proposed hybrid technique. While applying the proposed hybrid technique, the cost function should be minimized and reduced the losses of the system also. Before that, the bus data, line data, number of DG units, power flow limits is given as the input of the proposed hybrid technique. After that, the multi-objective function is achieved. The overall working process of the proposed method based OPF issues is illustrated in the Fig. 2. It shows that the detailed process of the computational procedure for optimal allocation of multiple DG units.

\subsection{Fuzzy logic controller}

The modeling approach used by FLC is similar to many error minimization techniques. Generally, FLC model has four layers such as fuzzifier, inference engine, knowledge based and defuzzifier is hypothesized. Next the input/ output the data is gathered for training the proposed

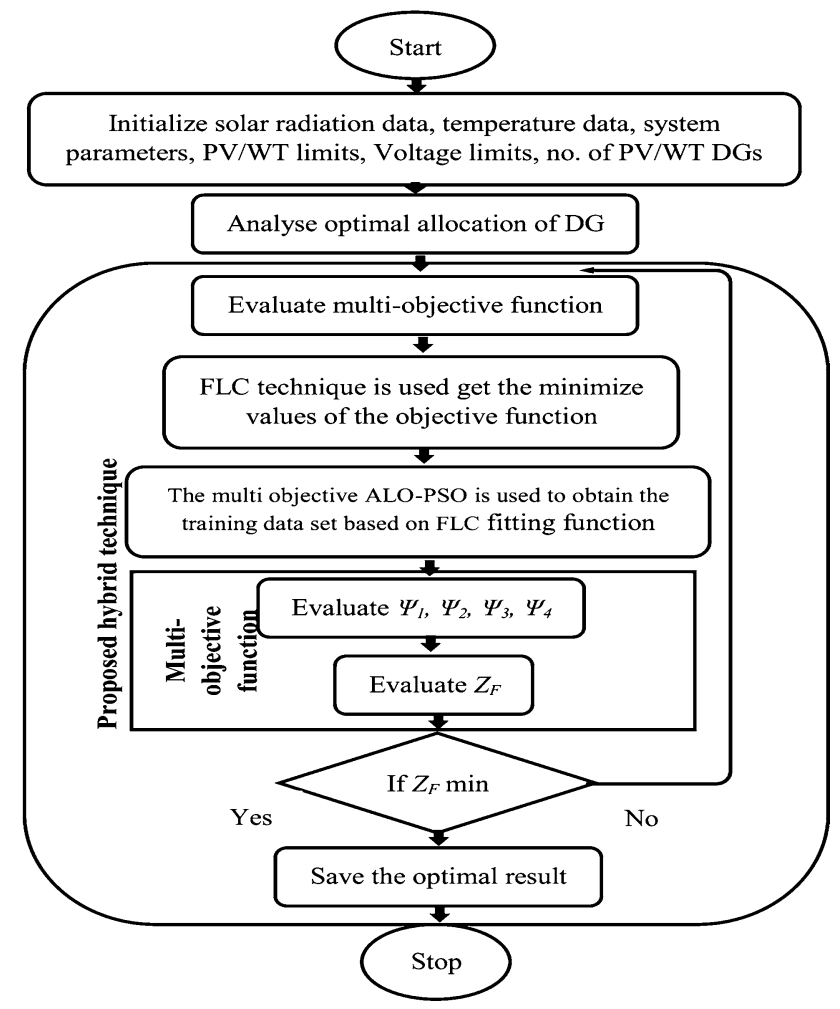

Fig. 2 Flowchart of overall Working process of proposed hybrid technique

system [23]. FLC can use to train the ALO-PSO to emulate the training data presented to it by modifying the parameters according to a chosen error criterion. The controller has been trained for all kinds of real and reactive power flow, power losses, power factor and enhancement voltage stability in the PV and WT based DG system. These changes allow the controller to achieve better when operating in strong control.

Step 1: Definition of the input/output characteristics. The first step is called knowledge, which is for selecting the position of fuzzy membership functions and choosing the number of inputs and outputs. In this work, the FLC represents line data, bus data, real and reactive power, line flow limits for the PV and WT system.

Step 2: Fuzzification structure.

The second step represents the inputs with convenient linguistic value by analyzing every input into a group. This step knows a unique MF label for example, "big", "medium" or "small". Thus, the FLC depends on the number of MFs used in the linguistic label. The MFs of the control the real and reactive power and allocate the WT and PV based DG system are defined as trapezoidal and triangular MFs.

Step 3: Inference Engine formation. 
The third stage explains how the fuzzy logic speed controller makes decision for WT and PV based DG system based on control rules and linguistic terms. In general, inference systems have two types, namely, Mamdani and Takagi-Sugeno [24].

Step 4: Defuzzification process converts fuzzy values into the crisp values.

Defuzzification is the final step in the fuzzy logic controller. This process generates the output values from the controller as a crisp value. Moreover, this process contains the important part of adjustment as well as the crisp value control of the output membership functions. However, these MFs are needed to select the number of the MFs and boundaries. Each MF must be suitable to give the optimized result through trial-and-error method.

\subsection{The ALO for optimal allocation of DG}

The ALO algorithm for selecting optimal training dataset has been briefly explained by following section.

\subsubsection{The random placements of the ant's and ant-Lion's}

The random placements of the ant's and ant-Lion's are represented in $M_{\text {ant-lion, }} M_{\text {ant }}$ [25],

$$
\begin{gathered}
M_{\text {ant-lion }}=\left(\begin{array}{cccc}
A L_{1,1} & A L_{1,2} & \ldots & A L_{1, n} \\
A L_{2,1} & A L_{2,2} & \ldots & A L_{2, d} \\
\vdots & \vdots & \vdots & \vdots \\
A L_{n, 1} & A L_{n, 2} & \ldots & A L_{n, d}
\end{array}\right) \\
M_{\text {ant }}=\left(\begin{array}{cccc}
A_{1,1} & A_{1,2} & \ldots & A_{1, n} \\
A_{2,1} & A_{2,2} & \ldots & A_{2, d} \\
\vdots & \vdots & \vdots & \vdots \\
A_{n, 1} & A_{n, 2} & \ldots & A_{n, d}
\end{array}\right)
\end{gathered}
$$

Fitness function of every ant-lion and ant have been given in the matrix $M_{O A L}, M_{O A}[25]$ and function $f$ is given as,

$$
\begin{gathered}
\left.M_{O A L}=\left(\begin{array}{c}
f\left(\left[A L_{1,1}, A L_{1,2}, \ldots, A L_{1, n}\right]\right. \\
f\left(\left[A L_{2,1}, A L_{2,2}, \ldots, A L_{2, d}\right]\right. \\
\vdots \\
f\left(\left[A L_{n, 1}, A L_{n, 2}, \ldots, A L_{n, d}\right]\right.
\end{array}\right]\right) \\
M_{O A}=\left(\begin{array}{c}
f\left(\left[A_{1,1}, A_{1,2}, \ldots, A_{1, n}\right]\right) \\
f\left(\left[A_{2,1}, A_{2,2}, \ldots, A_{2, d}\right]\right. \\
\vdots \\
f\left(\left[A_{n, 1}, A_{n, 2}, \ldots, A_{n, d}\right]\right)
\end{array}\right)
\end{gathered}
$$

\subsubsection{Movement of ants randomly}

For food search all the ants allowed to move randomly. This ants movement [25] is given by (24),

$X(t)=\left[\begin{array}{l}0, \operatorname{cumsum}\left(2 r\left(t_{1}\right)-1\right), \operatorname{cumsum}\left(2 r\left(t_{2}\right)-1\right), \\ \ldots, \operatorname{cumsum}\left(2 r\left(t_{n}\right)-1\right)\end{array}\right]$

Here, cumsum is total sum, $n$ signifies as total number of ants, $t$ described as random walk step and generation of random number denoted with $r(t)$ [25], rand is a random number produced with uniform distribution in $[0,1]$,

$r(t)=\left\{\begin{array}{l}1 \text { if rand }>0.5 \\ 0 \text { if rand } \leq 0.5\end{array}\right.$

The ant's position will be updated as [25],

$X_{i}^{t}=\frac{\left(X_{i}^{t}-p_{i}\right)\left(s_{i}-r_{i}^{t}\right)}{s_{i}^{t}-p_{i}}+r_{t}$

Here, $q_{i}$ and $p_{i}$ are upper and lower values of ant's random walk, $r_{i}^{t}$ and $s_{i}^{t}$ represents lower and higher $m$ th variable at $t$ th iteration.

\subsubsection{Ants trapping}

Ant's random walks are affected by traps of ant lion's traps given by [25].

$c_{m}^{t}=A n t-\operatorname{lion}_{n}^{t}+c^{t}$

$d_{m}^{t}=A n t-\operatorname{lion}_{n}^{t}+d^{t}$

\subsubsection{Trap construction}

This ALO is to select ant-lions by using roulette wheel operator to select ant lions depends on ant-lion fitness [25].

\subsubsection{Ants sliding into pits}

Ants sliding into pits represented by [25],

$c^{t}=\frac{c^{t}}{l}$

$d^{t}=\frac{d^{t}}{l}$ 
Here, $I=10^{w} t / s, t$ is present iteration, $S$ is the total iterations and $w$ is a constant, its value is given by [25],

$w=\left\{\begin{array}{r}2 \text { if } t>0.1 S \\ 3 \text { if } t>0.5 S \\ 4 \text { if } t>0.75 S \\ 5 \text { if } t>0.9 S \\ 6 \text { if } t>0.95 S\end{array}\right.$

\subsubsection{Prey catching and pit reconstruction}

Ant-lions now update their location to hold the new prey. This is represented by [25],

Ant $-\operatorname{lion}_{n}^{t}=A n t_{m}^{t}$ if $f\left(A n t_{m}^{t}\right)>f\left(A n t-\operatorname{lion}_{j}^{t}\right)$

\subsubsection{Elitism}

Here each ant is expected to associate with an ant-lion by Roulette wheel is represented by [25],

Ant $^{t}=\frac{R_{A}^{t}+R_{B}^{t}}{2}$

Here, at $t$ th iteration around the elite and selected ant lion are $R_{B}, R_{A}$. From all the above it is clear that ant's random walk is highly constrained around the elite ant-lions and as a significance, this approach faces poor examination. It is a well-known fact that, the efficiency of metaheuristic approach is highly depends on appropriate stability between exploitation and exploration.

PSO has been employed to improve the performance of the ALO, with the help of updating the elitism phase of the ALO. PSO is a stochastic population-based metaheuristic to solve continuous optimization problems. The main idea of the metaheuristic came from the observation of behavior of natural organisms to find food. PSO works with a swarm of particles. Each particle is a solution to a problem in the decision space and has two characteristic: its own position and velocity. The position represents the current values in the solution; the velocity defines the direction and the distance to optimize the position at next iteration. For each particle $i$ its own past best position $p$ best $i$ and the entire swarm's best overall position $G$ are remembered [26]. In basic PSO the velocity and position of each particle are updated in the following Eqs. (34) and (35),

$$
\begin{aligned}
& v_{i}(k+1)=w v_{i}(k)+\rho_{1} c_{1}\left(p_{i}^{\text {best }}-X_{i}(k)\right)+\rho_{2} c_{2}\left(G X_{i}(k)\right) \\
& X_{i}(k+1)=X_{i}(k)+v_{i}(k+1)
\end{aligned}
$$

Where, $i$ is a particle index, $k$ is an iteration number, $v_{i}(k)$ is velocity, $X_{i}(k)$ is the position of particle $i$ at iteration $k$, $P_{i}^{\text {best }}$ is the best position found by particle $i$ (personal best), $G$ is the best position found by the swarm (global best, best of personal bests), $w$ is an inertia coefficient, $\left(\rho_{1}, \rho_{2}\right)$ are random numbers in $[0,1]$ interval, $c_{1}, c_{2}$ are positive constants representing the factors of particle's attraction toward its own best position or toward the swarm's best position [27].

Over other optimization techniques, PSO has distinct advantages. It is simple in concept, ease in implementation, and efficient in computation in terms of both memory requirements and speed. For capacitor allocation problem each particle $i$ corresponds to a capacitor, the position $X_{i}(k)$ is a vector with components $X_{i j}(k)$ corresponded to a voltage magnitude at bus $j$ and capacitor $i$. The values of all the parameters such as the size of a swarm, inertia coefficient, factors of attraction have been defined by default in toolbox of MATLAB. After getting the optimal solution and it will update to the ALO parameters. Finally the ALO performances are improved with the help of PSO and to evaluate the effectiveness of the proposed technique.

After statement updating and all parameters were finalized, appropriate set of membership function and selection of rule base for FLC has done. To train FLC, the system was trained in off-line with IEEE-33 radial system. Detailed simulation analysis is worked out in the following section.

\section{Outcomes and discussions}

Application of projected hybrid scheme is carried on MATLAB. Result is tested in the IEEE 33 Radial scheme, which is having of branches 32 and buses 33 given in Fig. 3. Line data and load data of test bus network are illustrated in [28]. $12.66 \mathrm{kV}$ and $100 \mathrm{MVA}$ are base values and $3715 \mathrm{~kW}$ is total actual and $2300 \mathrm{kVAR}$ is total reactive power load. Power factor of load this network is $0.85 \mathrm{lag}, 0.85$ lead and Unity. For the implementation purpose, proposed hybrid technique and conventional scheme parameters are given in Table 1.

The result is estimated from the proposed hybrid technique and then it compares with the existing techniques with the parameters of the power losses reduction, operational cost and VSI then the comparison parameters are estimated from PSO and ALO and proposed hybrid technique. The proposed techniques is hybrid optimization technique, which is stands with the FLC technique and ALO-PSO algorithm, the testing is made up of 10 population sizes and it compares with the traditional techniques to reach the better solution in the proposed hybrid technique. For the hybrid technique has taken the maximum random generation is 100 . The following case studies were 
Fig. 3 IEEE 33 test bus

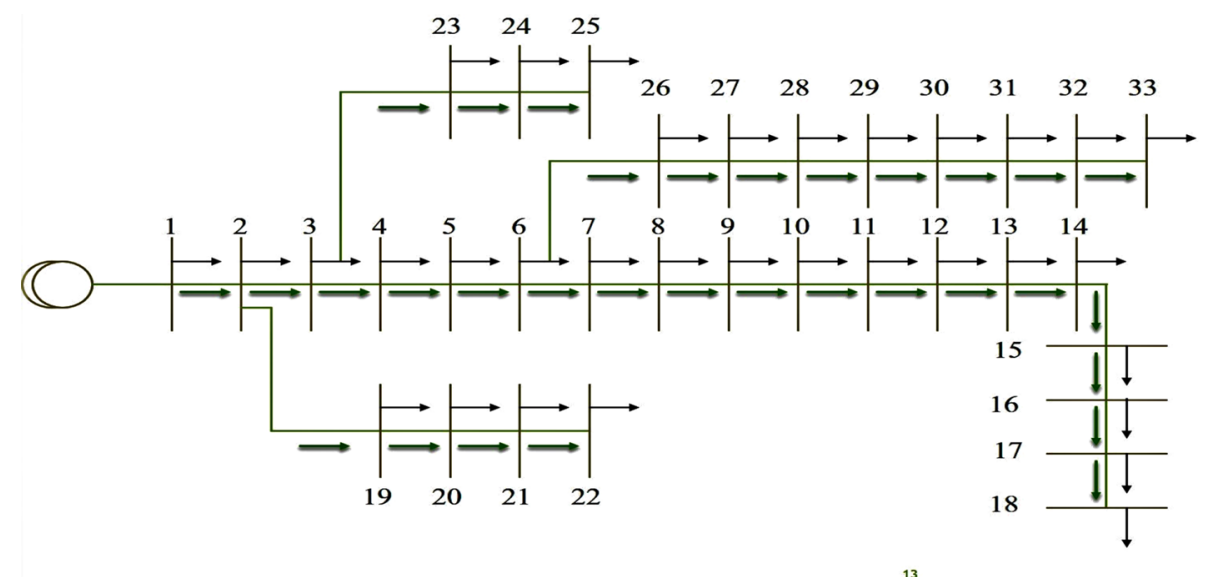

Table 1 Implementation parameters of projected technique

\begin{tabular}{lcc}
\hline Description & Algorithms & Values \\
\hline Ant lion pits & ALO & 3 \\
Number of Ant lions & 10 \\
Max iteration & & 100 \\
\hline
\end{tabular}

carried out to get reducing optimal allocation WT, PV bases DGs. In this study there are different cases, first one is the 33 bus system without PV and WT DGs, second one is with 2-PVs and 2-WT DGs with power factor of unit, third one is with 2-PVs and 2-WT DGs with power factor of $0.85 \mathrm{lag}$ and the fourth one is with 2-PVs and 2-WT DGs with power factor of 0.85 lead and finally optimal allocation of capacitors using proposed technique. Firstly, the discussion about the PV and DGs are without condition in 33 bus system.

\section{1st Case: without DGs}

This section describes the analysis of optimal allocation and sizing problems are optimized in without WT, and PV based DGs at normal condition. Table 2 presents outcomes of base case.

\section{2nd Case: with 2-WTs, and 2-PVs based DGs with unity power factor}

In this study the maximum capacity of 2-PV DG units is $70 \%$ of total power demand of test system. The total reactive and actual power loss after 2-PV DGs with unity power factor placement are 64.75 kVAr and $90.98 \mathrm{~kW}$ respectively and reduction of real and reactive powers are $51.59 \%$ and $54.93 \%$ respectively with minimum value of voltage enhanced from 0.9040 to 0.92337 p.u. 2-PV DGs are optimally placed at multiple buses with capacity of $385.0456 \mathrm{~kW}$ at 32 bus and $2154 \mathrm{~kW}$ at 7 bus. The minimum VSI enhanced from 0.69526 to 0.84153 p.u. TOC of system is also reduced from $\$ 19382.5701$ to $\$ 12062.8373$. The results are presented in Table 3. It is clear that projected hybrid technique considerably minimize the power loss and voltage is enhanced.

Here in this scenario type-1, type- 2 and type-3 WT and PVs are sited simultaneously in the network.

Scenario: Simultaneous placement of type-1, type-2 and type-3 WT and PV.

If a type- 1 and a type-3 WT and PV are sited together at various optimal positions, hence the losses further minimized when compared with type- 1 and type- 2 WT and PV placement and type- 2 and type- 3 considered. The outcomes attained for concurrent location of a type- 2 and a type-3 WT and PV are given in Table 4.

In this study the maximum capacity of 2-WT DG units are $70 \%$ of total power demand of test system. The total reactive and actual power loss after 2-WT DGs with unity
Table 2 Load demands, power losses, TOC and minimum at normal condition for IEEE 33-Bus system

\begin{tabular}{llllll}
\hline Parameters & $\begin{array}{l}\text { Active } \\
\text { power (kW) }\end{array}$ & $\begin{array}{l}\text { Reactive } \\
\text { power (kVAr) }\end{array}$ & VSI (p.u) & TOC (\$) & Minimum voltage (p.u) \\
\hline Load demands & 3715 & 2300 & 0.69526 & 19382.5701 & 0.9040 \\
Normal power loss & 201.8 & 134.6413 & & & \\
\hline
\end{tabular}




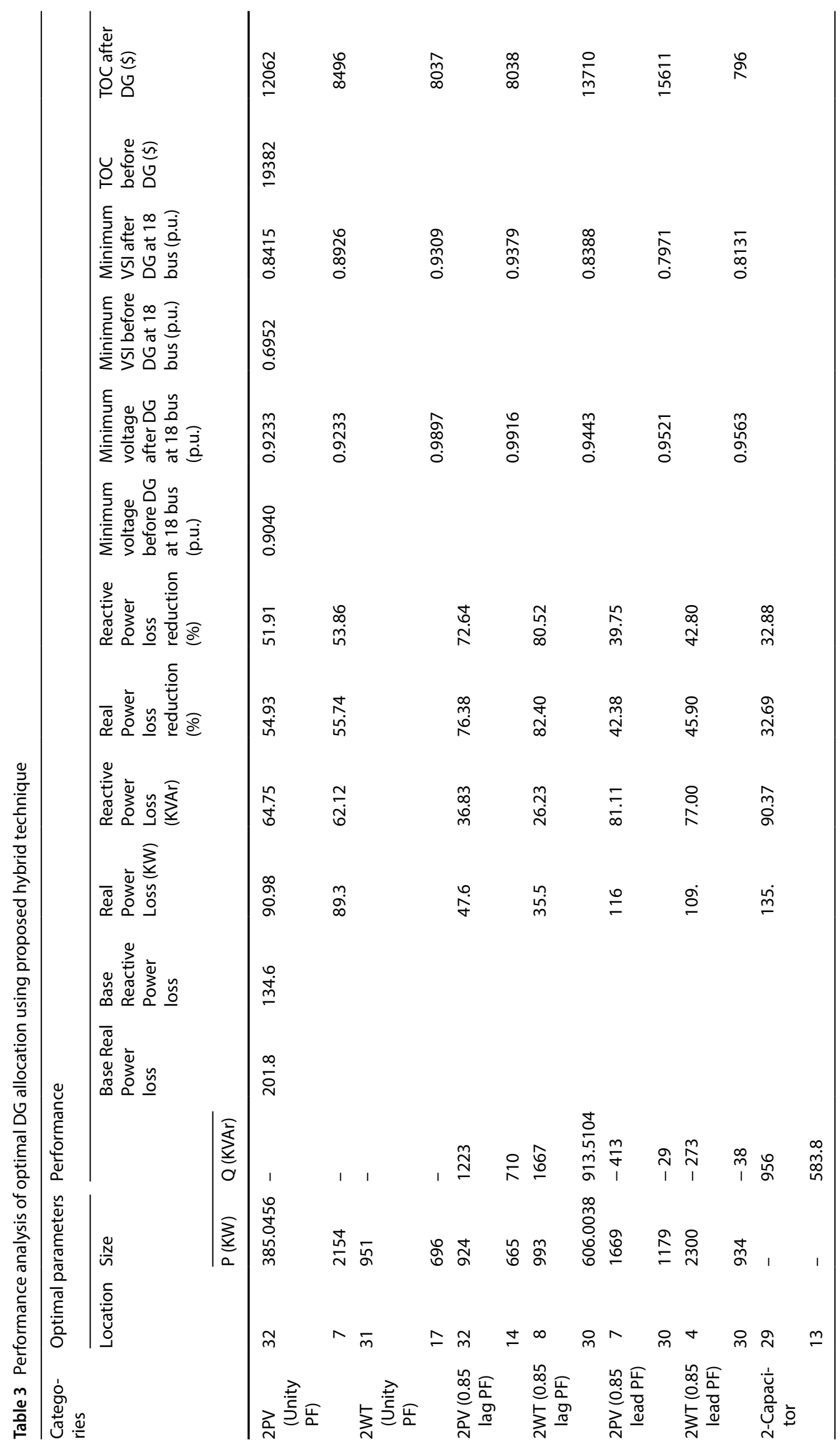


Table 4 Simultaneous type-1, type-2 and type-3 PV and WT placement using proposed hybrid technique

\begin{tabular}{|c|c|c|c|c|c|c|c|c|}
\hline \multirow[t]{3}{*}{ Analytical approach } & \multicolumn{6}{|c|}{ Capacity } & \multirow{2}{*}{\multicolumn{2}{|c|}{ Losses in kW }} \\
\hline & \multirow[t]{2}{*}{ Bus no } & \multicolumn{2}{|l|}{ PV } & \multicolumn{3}{|l|}{ WIND } & & \\
\hline & & kW & kVAr & $\mathrm{kW}$ & kVAr & $\mathrm{PF}$ & PL & QL \\
\hline \multirow[t]{2}{*}{ Simultaneous type- 1 and type-3 DG placement } & 32 & 793 & - & - & - & - & 37.3361 & 27.0355 \\
\hline & 9 & - & - & 1156.5784 & 1227.275 & 0.85 lag & & \\
\hline \multirow{2}{*}{ Simultaneous type- 1 and type-2 DG placement } & 13 & 964 & - & - & & - & 77.6564 & 48.1984 \\
\hline & 30 & - & - & - & 599 & - & & \\
\hline \multirow[t]{2}{*}{ Simultaneous type- 2 and type- 3 DG placement } & 29 & - & - & 1115.754 & 1183 & $0.85 \mathrm{lag}$ & 57.6498 & 38.3879 \\
\hline & 30 & - & - & - & 67 & - & & \\
\hline
\end{tabular}

power factor placement are $62.12 \mathrm{kVAr}$ and $89.3 \mathrm{~kW}$ respectively and reduction of real and reactive powers are $53.86 \%$ and $55.74 \%$ respectively with minimum value of voltage enhanced from 0.9040 to 0.92337 p.u. 2-WT DGs are optimally placed at multiple buses with capacity of $951 \mathrm{~kW}$ at 31 bus and $696 \mathrm{~kW}$ at 17 bus. The minimum VSI enhanced from 0.69526 to 0.89264 p.u. TOC of system is also reduced from $\$ 19382.5701$ to $\$ 8496.0656$. The results are presented in Table 3. It is clear that projected hybrid technique considerably minimize the power loss and voltage is enhanced.

\section{3rd Case: with 2-WT, and 2-PV based DGs with 0.85 lagging power factor}

In this study the maximum capacity of 2-PV DG units are $70 \%$ of total power demand of test system. The total real and reactive power loss after 2-PV DGs with 0.85 lagging power factor placement is $47.4 \mathrm{~kW}$ and $36.86 \mathrm{kVAr}$ respectively and the minimization of the reactive and real powers are $76.38 \%$ and $72.64 \%$ respectively with minimum value voltage enhanced from 0.9040 to 0.98979 p.u. The 2-PV DGs are optimally placed in multiple buses with capacity of $924 \mathrm{~kW}$ and $1223 \mathrm{kVAr}$ at 32 bus and $665 \mathrm{~kW}$ and $710 \mathrm{kVAr}$ at 14 bus. The minimum VSI enhanced from 0.69526 to 0.93093 p.u. TOC of system is also reduced from $\$ 19382.5701$ to $\$ 8037.6482$. The results are presented in Table 3. It is clear that projected hybrid technique considerably minimize the power loss and voltage is enhanced.

In this study the maximum capacity of 2-WT DG units are $70 \%$ of total power demand of test system. The total reactive and actual power loss after 2-WT DGs with 0.85 lagging power factor placement is $26.23 \mathrm{kVAr}$ and $35.5 \mathrm{~kW}$ respectively and the minimization of the reactive and real powers are $80.52 \%$ and $82.40 \%$ respectively with minimum value voltage enhanced from 0.9040 to 0.99168 p.u. The 2-WT DGs are optimally placed in multiple buses with capacity of $993 \mathrm{~kW}$ and $1667 \mathrm{kVAr}$ at 8 bus and
$606.0038 \mathrm{~kW}$ and $913.5104 \mathrm{kVAr}$ at 30 bus. The minimum VSI enhanced from 0.69526 to 0.9397 p.u. TOC of system is also reduced from $\$ 19382.5701$ to $\$ 8038.9$. The results are presented in Table 3. It is clear that projected hybrid technique considerably minimize the power loss and voltage is enhanced.

\section{4th Case: with 2-WT, and 2-PV based DGs with 0.85 leading power factor}

In this study the maximum capacity of 2-PV DG units is $70 \%$ of total power demand of test system. The total reactive and actual power loss after 2-PV DGs with 0.85 leading power factor placement is $81.1 \mathrm{kVAr}$ and $116 \mathrm{~kW}$ respectively and the minimization of the reactive and real powers are $39.75 \%$ and $42.38 \%$ respectively with minimum value voltage enhanced from 0.9040 to 0.96437 p.u. The 2-PV DGs are optimally placed in multiple buses with capacity of $1669 \mathrm{~kW}$ and $-416 \mathrm{kVAr}$ at 7 bus and $1179 \mathrm{~kW}$ and $-29 \mathrm{kVAr}$ at 30 bus. The minimum VSI enhanced from 0.69526 to 0.83882 p.u. TOC of system is also reduced from $\$ 19382.5701$ to $\$ 137100.0273$. The results are presented in Table 3. It is clear that projected hybrid technique considerably minimize the power loss and voltage is enhanced.

In this study the maximum capacity of 2-WT DG units are $70 \%$ of total power demand of test system. The total reactive and actual power loss after 2-WT DGs with 0.85 leading power factor placement is $77 \mathrm{kVAr}$ and $109 \mathrm{~kW}$ respectively and the minimization of the reactive and real powers are $42.80 \%$ and $45.90 \%$ respectively with minimum value voltage enhanced from 0.9040 to 0.95214 p.u. The 2-WT DGs are optimally placed in multiple buses with capacity of $2300 \mathrm{~kW}$ and $-273 \mathrm{kVAr}$ at 4 bus and $934 \mathrm{~kW}$ and $-38 \mathrm{kVAr}$ at 30 bus. The minimum VSI enhanced from 0.69526 to 0.79711 p.u. TOC of system is also reduced from $\$ 19382.5701$ to $\$ 15611.3215$. The results are presented in Table 3. It is clear that projected hybrid techniques considerably minimize the power loss and voltage is enhanced. 


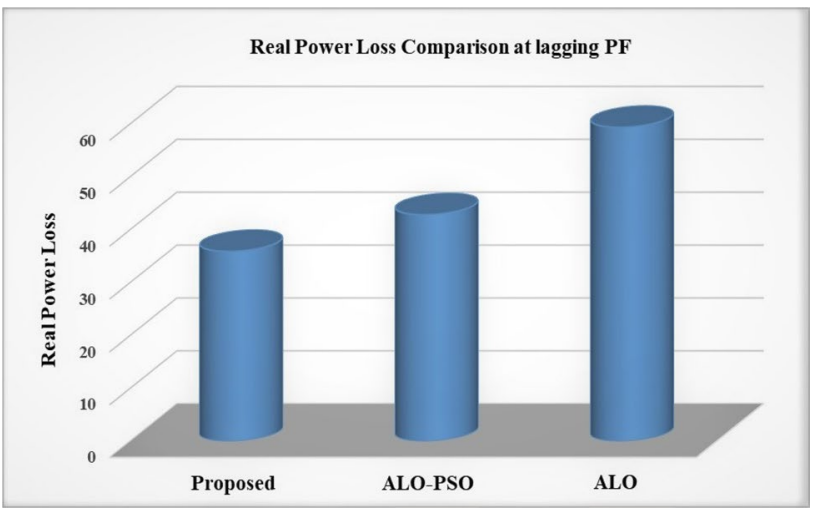

Fig. 4 Comparative analysis of real power loss at 0.85 lagging pf

\section{5th Case: size and optimal allocation of 2-capacitors using proposed hybrid technique}

In this study the maximum capacity of Capacitor units is $70 \%$ of the total power demand of test system. The total reactive and real power loss after 2-capacitors placement is $90.37 \mathrm{kVAr}$ and $135 \mathrm{~kW}$ respectively and the minimization of the reactive and real powers are $32.88 \%$ and $32.69 \%$ respectively with minimum value of voltage enhanced from 0.9040 to 0.95639 p.u. The 2-capacitors are optimally placed in multiple buses with capacity of $956 \mathrm{kVAr}$ at 29 bus and $583.8713 \mathrm{kVAr}$ at 13 bus. The minimum VSI enhanced from 0.69526 to 0.81313 p.u. TOC of system is also reduced from $\$ 19382.5701$ to $\$ 7964.4959$. The results are presented in Table 3. It is clear that projected hybrid technique considerably minimizes the power loss and voltage is enhanced.

\subsection{Comparative analysis}

The proposed hybrid FLC technique have been examined for solving IEEE-33 test scheme with testing for various studies. The outcomes of projected hybrid FLC technique also evaluated with the outcomes found from ALO algorithm and ALO-PSO method.

Figures $4,5,6,7,8,9,10,11,12$ and 13 presents outcomes in graphical format. With the proposed method actual and reactive power losses reduction, TOC reduction, minimum value of voltage improvement and the minimum VSI improvements are $82.22 \%, 80.32 \%, 8138.9$ $\$, 0.98168$ and 0.9279 respectively. The ALO and ALOPSO methods power losses reduction, total operational cost and voltage stability index values are $78 \%, 70 \%$, $70 \%, 60 \%, 10,000 \$, 12,000 \$, 0.965,0.969$ respectively.

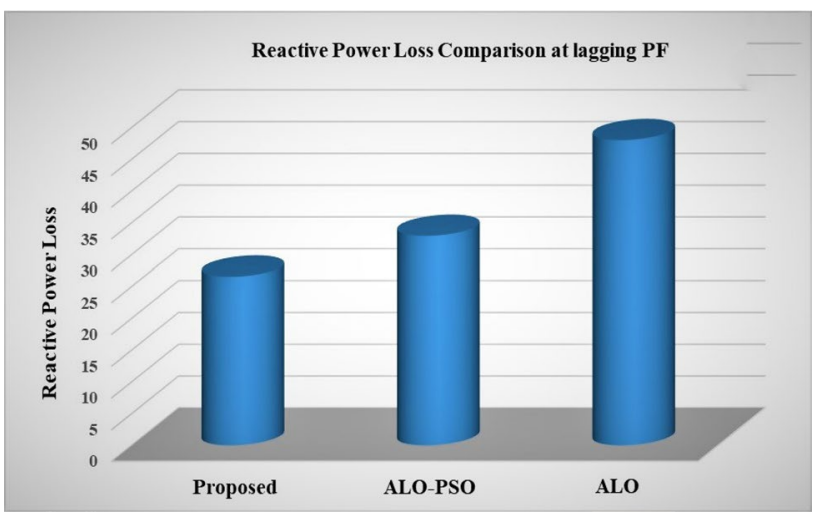

Fig. 5 Comparative analysis of reactive power loss at 0.85 lagging pf

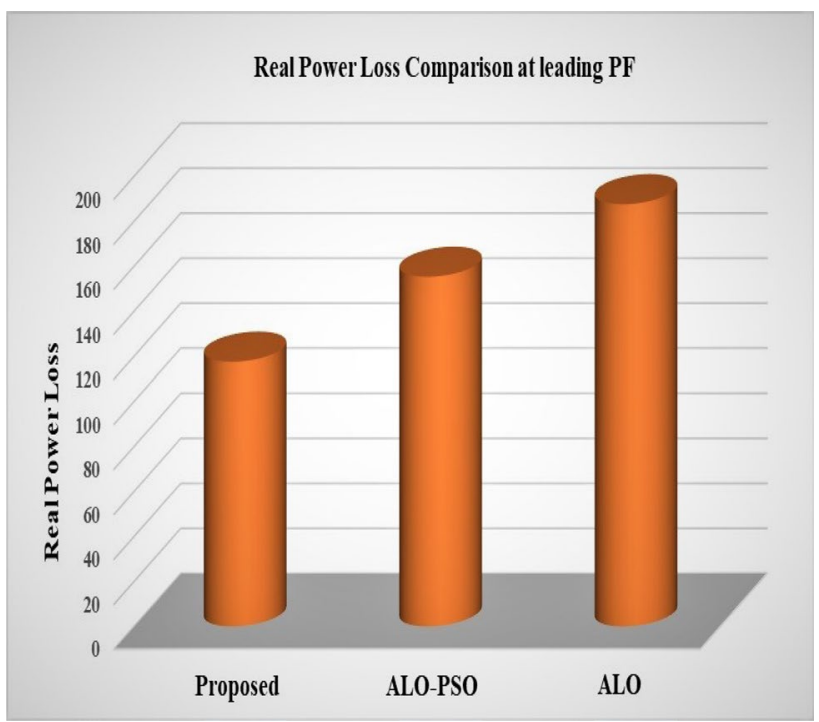

Fig. 6 Comparative analysis of real power loss at 0.85 leading pf

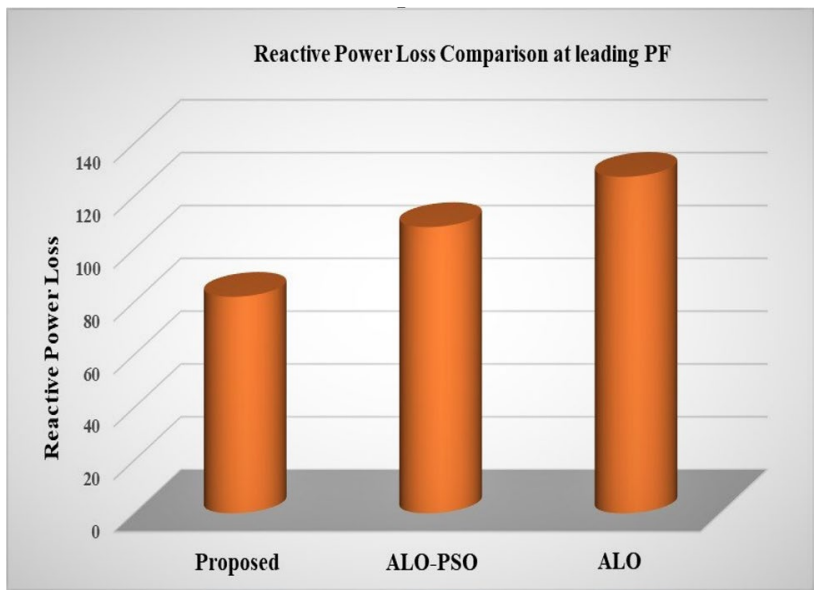

Fig. 7 Comparative analysis of reactive power loss at 0.85 leading pf 


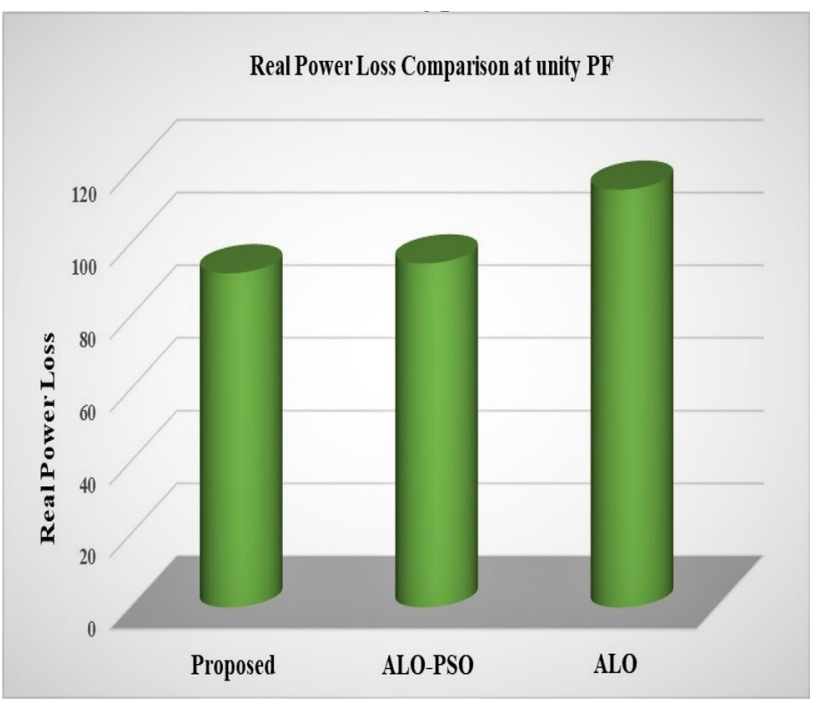

Fig. 8 Comparative analysis of real power loss at unity pf

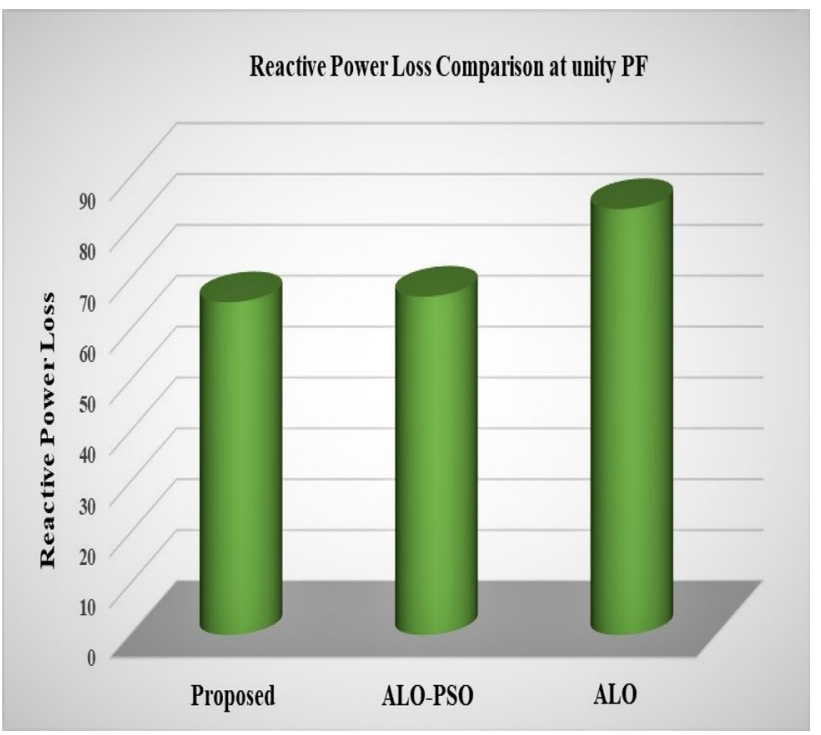

Fig. 9 Comparative analysis of reactive power loss at unity pf

\section{Conclusion}

This research proposed an efficient technique to solve optimal DG allocation problems of power system. Initially, the IEEE 33 test bus was considered for optimal load flow issues. In addition, the WT, and PV based DGs are utilized and the corresponding operating cost index and VSI are also determined. Formulated optimal

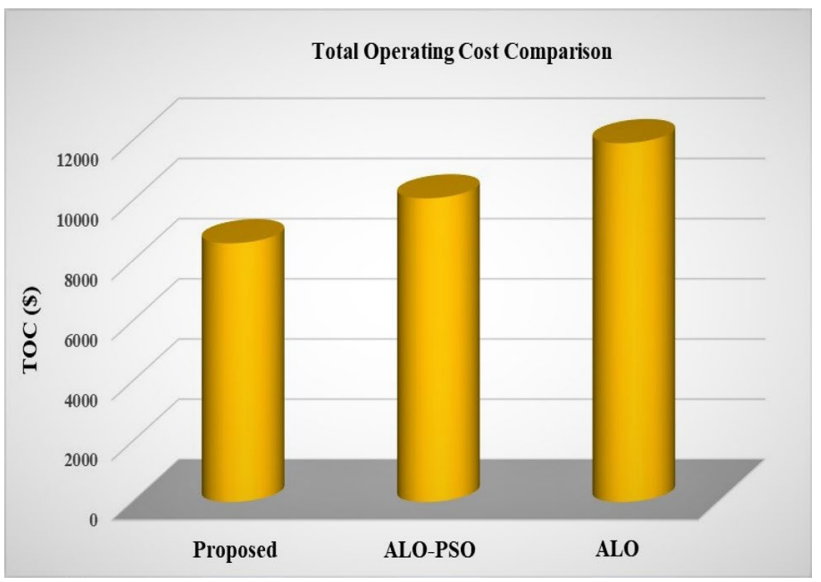

Fig. 10 Comparative analysis of total operating cost

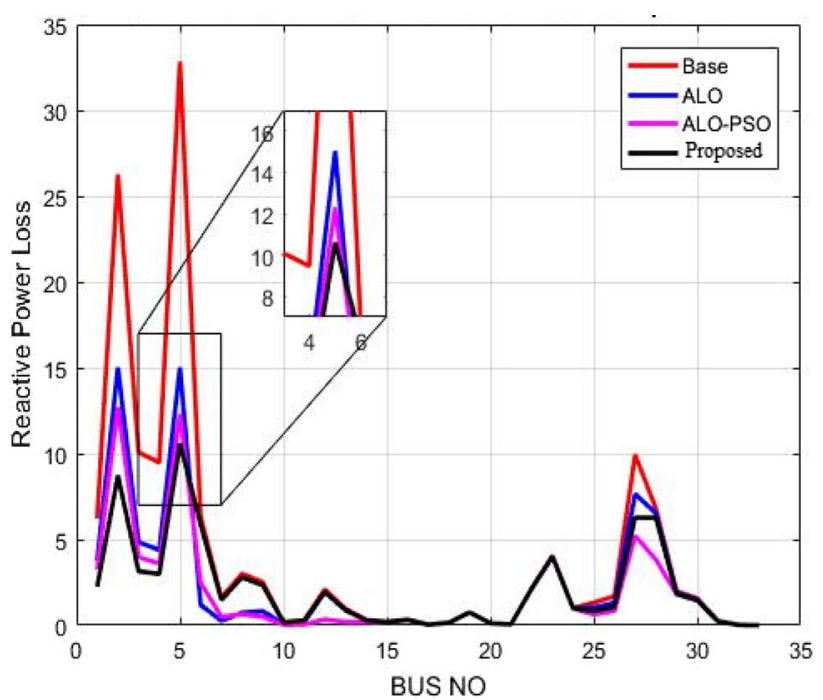

Fig. 11 Comparative analysis of reactive power loss at every bus

power flow issues have been resolved while satisfying network inequality and equality constraints. Projected hybrid technique has verified its efficiency by starting the iteration procedure with a decent primary value and reaches an absolute finest value in minimum number of iterations. Hence, it is decided that the ultimate enhancement in loss minimization, improvement in VSI with together location of WT \& PV. This projected hybrid technique will work on any type of radial distribution network. 


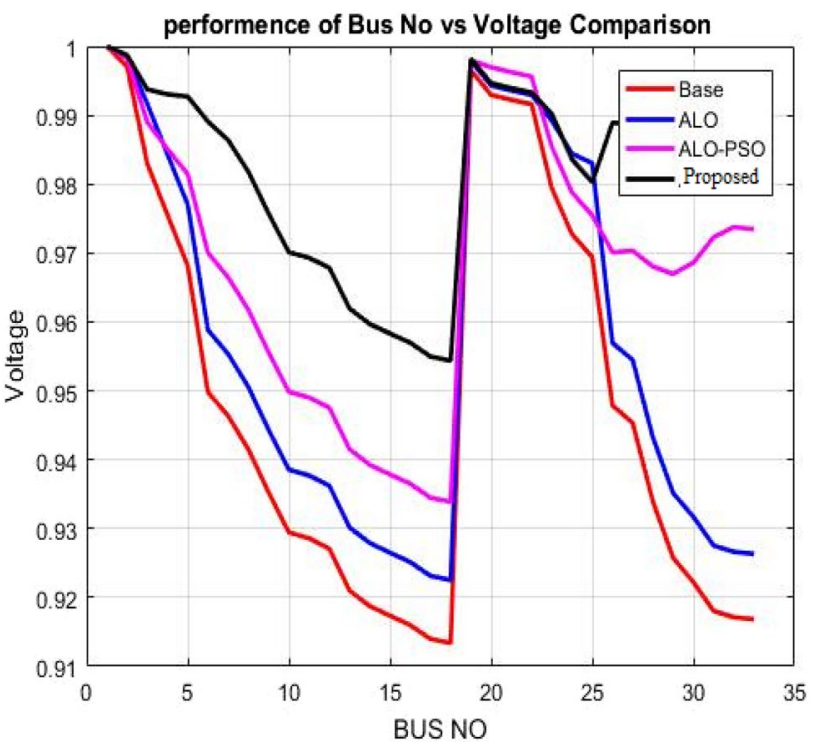

Fig. 12 Comparative analysis of voltage at every bus

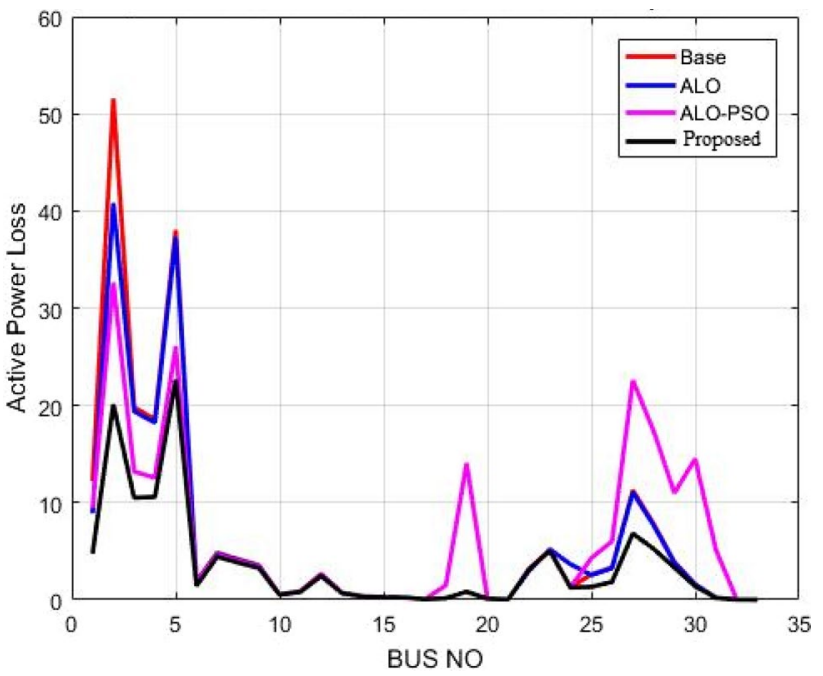

Fig. 13 Comparative analysis of active power loss at every bus

\section{Compliance with ethical standards}

Conflict of interest The authors report no conflicts of interest. The authors alone are responsible for the content and writing of this article.

\section{References}

1. M Sarwar, AS Siddiqui, Z Jaffery and IA Quadri (2018) Optimal placement of distributed generation for congestion management: a comparative study. In: 2 nd IEEE International conference on power electronics, intelligent control and energy systems
(ICPEICES) vol 53, pp 229-233 https://doi.org/10.1109/icpei ces.2018.8897282

2. Kansal S, Kumar V, Tyagi B (2016) Hybrid approach for optimal placement of multiple DGs of multiple types in distribution networks. Int J Electr Power Energy Syst 75:226-235. https://doi. org/10.1016/j.ijepes.2015.09.002

3. Sultana U, Azhar B, Khairuddin MM, Aman ASM, Zareen N (2016) A review of optimum DG placement based on minimization of power losses and voltage stability enhancement of distribution system. Int J Renewable Sustain Energy Rev 63:363-378. https ://doi.org/10.1016/j.rser.2016.05.056

4. Bazrafshan M, Gatsis N, Dall'Anese E (2019) Placement and sizing of inverter-based renewable systems in multi-phase distribution networks. IEEE Trans Power Syst 34(02):918-930. https:// doi.org/10.1109/TPWRS.2018.2871377

5. Nayanatara C, Baskaran J, Kothari DP (2016) Hybrid optimization implemented for distributed generation parameters in a power system network. Int J Electr Power Energy Syst 78:690-699. https://doi.org/10.1016/j.ijepes.2015.11.117

6. Rahmani-andebili M (2016) Distributed generation placement planning modeling feeder's failure rate and customer's load type. IEEE Trans Industr Electron 63(3):1598-1606. https://doi. org/10.1109/TIE.2015.2498902

7. Zhan H, Wang C, Wang Y, Yang X, Zhang X, Wu C, Chen Y (2016) Relay protection coordination integrated optimal placement and sizing of distributed generation sources in distribution networks. IEEE Trans Smart Grid 7(1):55-65. https://doi.org/10.1109/ TSG.2015.2420667

8. Mahipal B, Babu Naik G, Naresh Kumar Ch (2016) A novel method for determining optimal location and capacity of DG and capacitor in radial network using weight-improved particle swarm optimisation algorithm (WIPSO). Int J Adv Res Electr Electron Instrum Eng 5(5):3478-3485. https://doi.org/10.15662 /IJAREEIE.2016.0505004

9. Pereira BR, da Costa GRM, Contreras J, Mantovani JRS (2016) Optimal distributed generation and reactive power allocation in electrical distribution systems. IEEE Trans Sustain Energy 7(3):975-984. https://doi.org/10.1109/TSTE.2015.2512819

10. Hari Prasad C, Subbaramaiah K, Sujatha P (2019) Cost-beneft analysis for optimal DG placement in distribution systems by using elephant herding optimization algorithm. Renewables 6(2):1-12. https://doi.org/10.1186/s40807-019-0056-9

11. Wang C, Song G, Li P, Ji H, Zhao J, Wu J (2017) Optimal siting and sizing of soft open points in active electrical distribution networks. Int J Appl Energy 189:301-309. https://doi.org/10.1016/j. apenergy.2016.12.075

12. Mokgonyana L, Zhang J, Li H, Yihua Hu (2017) Optimal location and capacity planning for distributed generation with independent power production and self-generation. Int J Appl Energy 188:140-150. https://doi.org/10.1016/j.apene rgy.2016.11.125

13. A Selim, S Kamel and F Jurado (2018) Hybrid optimization technique for optimal placement of DG and D-STATCOM in distribution network. In: International middle east power systems conference (MEPCON), Cairo University, Egypt, vol 8. no. 18, pp 689-693. https://doi.org/10.1109/mepcon.2018.8635253

14. Ali ES, Abd Elazim SM, Abdelaziz AY (2017) Ant Lion optimization algorithm for optimal location and sizing of renewable distributed generations. Int J Renew Energy 101:1311-1324. https:// doi.org/10.1016/j.renene.2016.09.023

15. Babacan O, Torre W, Kleissl Jan (2017) Siting and sizing of distributed energy storage to mitigate voltage impact by solar PV in distribution systems. Int J Solar Energy 146:199-208. https:// doi.org/10.1016/j.solener.2017.02.047

16. El-Ela AAA, El-Sehiemy RA, Abbas AS (2018) Optimal placement and sizing of distributed generation and capacitor banks 
in distribution systems using water cycle algorithm. IEEE Syst J 99:1-8. https://doi.org/10.1109/JSYST.2018.2796847

17. Samala RK, Kotapuri MR (2018) Power loss reduction using distributed generation. Model, Meas Control A 91(3):104-113. https://doi.org/10.18280/mmc_a.910302

18. Prabha DR, Jayabarathi T (2016) Optimal placement and sizing of multiple distributed generating units in distribution networks by invasive weed optimization algorithm. Ain Shams Eng J 7(2):683-694. https://doi.org/10.1016/j.asej.2015.05.014

19. Samala RK, Kotapuri MR (2018) Optimal DG sizing and siting in radial system using hybridization of GSA and Firefly algorithms. Model, Meas Control A 91(2):77-82. https://doi.org/10.18280/ mmc_a. 910208

20. Kowsalya M (2014) Optimal size and siting of multiple distributed generators in distribution system use bacterial foraging optimization. Int J Swarm Evolut Comput 15:58-65. https://doi. org/10.1016/j.swevo.2013.12.001

21. Xu X, Xu Z, Lyu Xue, Li Jiayong (2018) Optimal SVC placement for maximizing photovoltaic hosting capacity in distribution network. IFAC-Papers OnLine 51(28):356-361. https://doi. org/10.1016/j.ifacol.2018.11.728

22. Hung DQ, Mithulananthan N (2013) Multiple distributed generator placement in primary distribution networks for loss reduction. IEEE Trans Industr Electron 60(4):1700-1708. https://doi. org/10.1109/TIE.2011.2112316

23. Pandiarajan K, Babulal CK (2016) Fuzzy harmony search algorithm based optimal power flow for power system security enhancement. Int J Electr Power Energy Syst 78:72-79. https:// doi.org/10.1016/j.ijepes.2015.11.053

24. Kim I (2017) Optimal distributed generation allocation for reactive power control. IET Gener Transm Distrib 11(6):1549-1556. https://doi.org/10.1049/iet-gtd.2016.1393

25. Samala RK, Kotapuri MR (2017) Multi distributed generation placement using ant-lion optimization. European J Electr Eng 5(6):253-267. https://doi.org/10.3166/ejee.19.253-267

26. Devi S, Geethanjali M (2014) Optimal location and sizing determination of distributed generation and DSTATCOM using particle swarm optimization algorithm. Int J Electr Power Energy Syst 62:562-570. https://doi.org/10.1016/j.ijepes.2014.05.015

27. Taher SA, Karimian A, Hasani M (2011) A new method for optimal location and sizing of capacitors in distorted distribution networks using PSO algorithm. Int J Simul Model Pract Theory 19:662-672. https://doi.org/10.1016/j.simpat.2010.09.001

28. Aderibole A, Zeineldin HH, Al Hosani M (2019) A Critical assessment of oscillatory modes in multi-microgrids comprising of synchronous and inverter-based distributed generation. IEEE Trans Smart Grid 10(3):3320-3330. https://doi.org/10.1109/ TSG.2018.2824330

Publisher's Note Springer Nature remains neutral with regard to jurisdictional claims in published maps and institutional affiliations. 